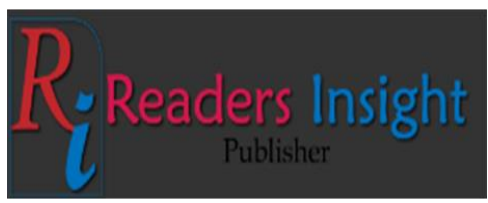

\title{
Study on the complications by chronic diabetes mellitus type II in Quetta Balochistan
}

\author{
Furqan Ahmed ${ }^{1 *}$, Mohsin Jamal Buzdar ${ }^{1}$, Alam Mengal ${ }^{2}$, Tahir Hameed ${ }^{1}$, Farhat Abbas ${ }^{1}$, Hafiza \\ Mehreen Tahir ${ }^{3}$ and Maryum Yousaf ${ }^{1}$.
}

${ }^{I}$ Center of Advanced Studies in Vaccinology and Biotechnology(CASVAB), University of Balochistan(UOB), Quetta, Pakistan

${ }^{2}$ Department of Microbiology, University of Balochistan (UOB), Quetta, Pakstan.

${ }^{3}$ Department of Biotechnology, Lahore College for Women University Lahore, Pakistan

${ }^{*}$ Corresponding author: ahmedfuki786@gmail.com

\section{Abstract}

Due to worl wide increase in Diabetes Mellitus, a rise community consciousness about influence of hyperglycemiea and its most common complications ike stroke, hypertension, nephropathy, amputation, neuropathy, cardiovascular and retinopathy etc is required. Awareness on adoption of good diet, physical activities as well as a healthy life-style is required for a healthy long life. Therefore, this study has been designed to investigate the burden of complications of chronic diabetes mellitus in diabetic patients of Quetta city, in order to improve the health status and life style of diabetic patients living in Quetta. Detail of all the complications caused by diabetes mellitus were collected from the patients and the bllood glucose was permormed by standard method. This study reaveled the frequency percentage of comorbidities in this order crdiovascular conditions $30 \%>$ neuropathy conditions $17.8 \%>$ Ocular conditions $14.8 \%>$ Nephropathy conditions $10.7 \%>$ Cerebrovascular $6.8 \%>$ Feet conditions $1.5 \%>$ Amputations $0.1 \%$. These results are in agreement to previous studies which showed the highest prevelance of cardiovascular diseases (CVDs) in diabetic patients with hyperglycemia, hypertension, smoking habits and unhealthy life styles.

Keywords: Complications, neeuropathy, nephropathy, CVD

\section{ARTICLE INFORMATION}

Received: 06.08.2019

Revised: 09.09.2019

Accepted: 30.09 .2019

DOI: $10.31580 /$ pjmls.v2i3.1126

\section{INTRODUCTION}

Diabetes Mellitus is rendered as a syndrome with heterogeneous group of diseases characterized by impaired glucose regulation as a consequence of chronic hyperglycemia [1]. There are three common types of diabetes mellitus; Type 1 is Insulin Dependent DM, Type 2 is Non-insulin Dependent Diabetes mellitus (DM) and Type 3 is gestational diabetes, which occurs sometimes in pregnancy: Dramatic increase in diabetes mellitus has adversely affected the health quality throughout the world [2]. International Diabetes Federation indicated that about 415 million people suffered from Diabetes Mellitus in 2015, which will rise to 642 million till 2040. In Pakistan, 1 in 11 adults are diabetic and 86,364 adults died due to diabetes [3].

Diabetes mellitus stays multifaceted long-lasting ailment allied with state of extraordinary blood glucose level, or else hyperglycemia, occurring from insufficiencies in insulin secretion, action otherwise both continuing metabolic inequity connected through this illness puts patients a high risk for long stretch macro also micro vascular difficulties, which if not on condition that with great quality maintenance, lead towards regular hospitalization besides impediment, counting raised risk intended for CVDs; cardiovascular diseases [4]. The most common complications in diabetic patients are stroke, hypertension, nephropathy, amputation, neuropathy, cardiovascular, retinopathy, impotency and skin lesions etc.

Retinopathy leads to blindness in diabetic patients in adults [5]. Longer diabetes duration and poorer glycemic and blood pressure control are strongly associated with Diabetic Retinopathy [6].

A prospective study indicated that apart from glycemic control the incidence of neuropathy is associated with potentially modifiable cardiovascular risk factors including a raised triglyceride level, body mass index, smoking and hypertension. Diabetes mellitus stays communal subordinate reason of hyperlipidaemia, predominantly, uncertainty glycaemic mechanism stays deprived, which in-turn remains significant menace influence intended atherosclerosis as well as coronary heart ailment [7].

Type 2 Diabetes Mellitus stays frequently connected with collection of numerous supplementary risk influences of cardiovascular diseases (CVD) for instance older age, insulin opposition, fatness, hyper tension, deprived glycemic prestige, 
variations in demagogic, clotting besides thrombolytic markers in addition towards dyslipidemia [8]. Bunching of numerous of these risk influences often manifests as metabolic set of symptoms that come first besides then remains by way of diabetes [9]. Diabetes stays sound-recognized sovereign risk issue intended for cardiovascular diseases (CVD). Associated by means of non-diabetic individuals, diabetic patients have 2 to 4 times greater than before risk intended for stroke as well as death from heart ailment [10]. Various co-variate risk influences of cardiovascular diseases (CVD) like smoking, physical inactivates, poor glycemic control, obesity as well as hypertension suggestive of high risk of forthcoming CVD. Therefore, it is need of time, to give awareness about diabetes and the complications related to it, so people can improve their life styles.

\section{METHODOLOGY}

The research was carried out in different sex groups, different age groups of different ethnic groups living in Quetta Balochistan, by collecting their blood samples with a questionnaier.

Equal samples of males and females were collected randomly for this purpose from different age groups. The demographic and behavioral risk factors were collected through face to face interviews, using an interview administered questionnaire. Each participant was questioned for age, sex, education, occupation, income, physical activity, alcohol consumption and smoking, cast, medication, type of diabetes and co morbidity.
Complications by chronic diabetes mellitus like eye diseases, heart diseases, kidney dis-function, neuropathy and muscular dystrophy were also inquired in the questionnaire.

Fasting blood glucose measurements were taken after an overnight fasting (>8h). These measurements were performed for all the participants using standard methods for glucose analysis and the results were recorded.

In order to calculate the body mass index (BMI) height and weight of different groups of patients were measured. Both weight, i-e, machines digital and analogue were used for the purpose. BMI was calculated by a formula weight in kilograms divided by height in meters square.

\section{Data Analysis}

The data was analyzed statistically by using computer package SPSS version 20. Frequency and percentages were computed to present all the categorical variables by mean and standard deviations. Analysis of Variance test will be performed by SPSS 20.

\section{RESULTS}

The blood glucose of all the diabetic patients was detrmined and cateogarized as shown in Figure 1.

Table 1 shows the prevelance and frequency percentage of complications related to chronic diabetes mellitus typell in diabetic patients of quetta city. 52\% diabetic patients were suffering from different types of pathological conditions conditions presented in Table 1.

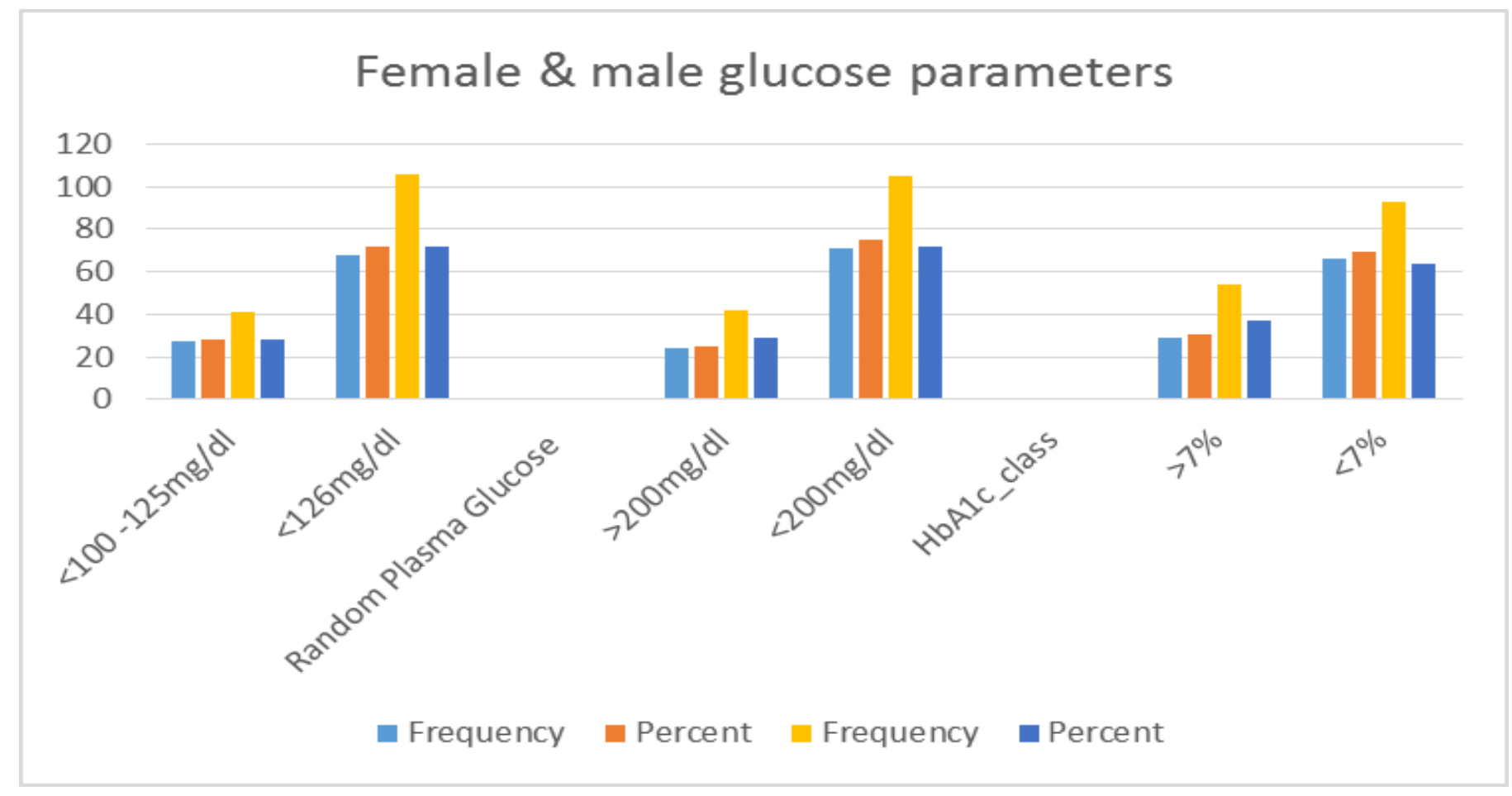

Fig 1. Female and male ADA \& NGSP recommended cut off values \& observed values

The ferquency percentage of data explore $30 \%$ occurance of cardiovascular conditions followed by $17.8 \%$ Neuropathy, $14.8 \%$ Ocular conditions, $10.7 \%$ Nephropathy conditions, $6.8 \%$ Cerebrovascular conditions $0.8 \%$ Foot diseases, $0.7 \%$ Foot ulcer and $0.1 \%$ Amputation. Among Cardiovascular conditions the frequency distribution of Angina was $10.6 \%$, Hypertension $8.5 \%$, chronic heart failure $3 \%$, heart diseases $1 \%$, infarction $0.7 \%$ and peripheral vascular diseases $0.4 \%$.
In Nephropathic conditions the most frequent condition was Microalbuminuria $7 \%$, which was followed by renal failure $1.9 \%$, Macroalbuminuria $0.8 \%$ and Renal hypofunction $0.3 \%$. The frequency percentage of Ocular conditiones involve Catract $8.6 \%$, Retinopathy $4.8 \%$ and Blindness $0.1 \%$.

In cerebrovascular conditions the frequency distribution of Transient ischemic disease (TIA) $3.6 \%$, was found greater than 
Stroke $2.4 \%$. Whereas the occurance of both stroke and TIA was $0.8 \%$.

Table 1. Complications relates to chronic diabetes II

\begin{tabular}{|c|c|c|}
\hline Categories by organ \& system & $\begin{array}{l}\text { No. of } \\
\text { case }\end{array}$ & $\%$ \\
\hline Cardiovascular conditions & 459 & 30.1 \\
\hline 1 cardio-condition & 381 & 25.0 \\
\hline Angina & 161 & 10.6 \\
\hline Hypertension & 130 & 8.5 \\
\hline Chronic heart failure & 45 & 3.0 \\
\hline Heart disease & 16 & 1.0 \\
\hline Infarction & 12 & 0.8 \\
\hline $\begin{array}{l}\text { Percutaneous transluminal coronary } \\
\text { angioplasty }\end{array}$ & 11 & 0.7 \\
\hline Peripheral vascular disease & 6 & 0.4 \\
\hline 2 cardio-conditions concurrently & 70 & 4.6 \\
\hline Angina + other cardio-conditions & 47 & 3.1 \\
\hline Other 2 cardio-conditions concurrently & 23 & 1.5 \\
\hline 3 cardio-conditions concurrently & 8 & 0.5 \\
\hline Angina + other cardio-conditions & 8 & 0.5 \\
\hline Cerebrovascular conditions & 103 & 6.8 \\
\hline 1 cerebro-condition & 91 & 6.0 \\
\hline Stroke & 37 & 2.4 \\
\hline Transient ischemic attack (TIA) & 54 & 3.6 \\
\hline Both stroke and TIA & 12 & 0.8 \\
\hline Neuropathy & 271 & 17.8 \\
\hline Nephropathy conditions & 162 & 10.7 \\
\hline 1 nephro-condition & 152 & 10.0 \\
\hline Microalbuminuria & 107 & 7.0 \\
\hline Macroalbuminuria & 12 & 0.8 \\
\hline Renal hypofunction & 4 & 0.3 \\
\hline Renal failure & 29 & 1.9 \\
\hline 2 nephro-condtions concurrently & 10 & 0.7 \\
\hline Microalbuminuria + other nephrocondition & 10 & 0.7 \\
\hline Ocular conditions & 225 & 14.8 \\
\hline 1 ocular condition & 205 & 13.5 \\
\hline Cataract & 131 & 8.6 \\
\hline Retinopathy & 73 & 4.8 \\
\hline Blindness & 1 & 0.1 \\
\hline 2 ocular conditions concurrently & 20 & 1.3 \\
\hline Cataract + Retionpathy & 19 & 1.2 \\
\hline Retionpathy + Blindness & 1 & 0.1 \\
\hline Foot diseases & 12 & 0.8 \\
\hline Foot ulcer & 10 & 0.7 \\
\hline Amputation(AMP) & 2 & 0.1 \\
\hline Single category & 465 & 30.5 \\
\hline 2 category concurrently & 234 & 15.4 \\
\hline 3 category concurrently & 74 & 4.9 \\
\hline 4 plus category concurrently & 19 & 1.3 \\
\hline Total & 792 & 52.0 \\
\hline
\end{tabular}

\section{DISCUSSION}

There are many physiological causes of complications related to diabetes like high blood pressure, smoking, elevated cholesterol levels, obesity and lack of regular exercise which can be controlled by a healthy life style. Overall, complications become less common and severe in diabetic patients with controlled blood glucose levels [11]. Hyperglycemia, coma, macrovascular disease, microangiopathy and immune dysfunction are common complications related to diabetes mellitus. Microangiopathy can affect all vital organs like heart, kidneys and brain, as well as eyes, nerves, lungs and locally gums and feet. Similar results were found in current study, about $52 \%$ of diabetic patients were having such comorbidities involving their vital organs like heart, kidneys, brain, feet and eyes.
International Diabetes Federation has estimated that numeral societies by way of diabetes global in 2015 stayed 415 million besides this remains estimated towards range 642 million in 2040. In 2015 diabetes stayed principal root of mortality, whereby 5 million individuals expired from diabetes also diabetes-related difficulties. Long-term macrovascular technical hitches of diabetes subsidize towards extraordinary morbidity also mortality allied with ailment with as many as $80 \%$ dying from some form of cardiovascular disease. In 2017, approximately (7.5 (5.3-10.9) million) Pakistanis had leading numeral adults aged 20-79 years with diabetes [3].

Cardio Vascular Diseases (CVD) stays main reason of expiry also disability in societies through diabetes. In young publics by means of type 1 diabetes (8-43 years), able to five people out of 1,000 expire since CVD each year, whereas between middlematured publics by type 2 diabetes existing great as well as intermediate revenue populations, fit for 27 entities out of 1,000 expire since CVD each year; a third of them expire as of stroke, in addition to quartier expire as of coronary artery disease [13].

In the present study it was observed that among all other comorbidities, the cardiovascular diseases were found highest in frequency $30.1 \%$, which shows the prevalence of CVDs amongall other comorbidities and its adverse effect on health of diabetic patients.

The prevelance of neuropathy was found at $2^{\text {nd }}$ number, which was followed by Ocular conditions. Nephropathy was found at $4^{\text {th }}$ number in comorbidities found in diabetic patients of Quetta city. If diabetic patients improve their eating and excersice habits they can control their sugar levels and can be at lower risk of these comorbidities.

As said by ADA as well as WHO Standard [12] in female also male patients of revision $(28.4 \%)$ besides $(27.9 \%)$ of patient standards intended for FPG stayed required, (71.6\%) and $(72.1 \%)$ of patient's high value for FPG. In female also male patients $(25.3 \%)$ and $(28.6 \%)$ of patient standards intended for RPG stayed required, $(74.7 \%)$ and $(71.4 \%)$ of patient's great rate intended for RPG. As stated by Recommendation of $(30.5 \%)$ and $(36.7 \%)$ of patient standards for $\mathrm{HbA} 1 \mathrm{c}$ stayed desirable, $(69.5 \%)$ and $(63.3 \%)$ of patient's high value intended for $\mathrm{HbA1c}$ [12]. Similar results were obtained in the current study for male and female daibetic patients.

The present study can create awareness of complication of diabetes in public of Quetta. Diabetic patients should reduce smoking control hyperglycemia, obesity and hypertension. They should increase physical activates and take care of their diet in order to get rid of these complications related to diabetes.

\section{Conclusion}

This study on the complications by chronic diabetes mellitus type II in diabetic patiesnt of Quetta Balochistan has reaveled the prevelance of comorbidities in this order crdiovascular conditions > neuropathy conditions > Ocular conditions > Nephropathy conditions $>$ Cerebrovascular $>$ Feet conditions $>$ Amputations.

\section{REFERENCES}

1. Koda-Kimble MA and Young LY. Applied Therapeutics: Clinical Use of Drugs: Springer. 2016.

2. Hwee W. Health-related Quality of Life in People with Diabetes Mellitus: Perspective from a Multiethnic Asian Population, PhD Thesis. 2005. 
3. IDF. International Diabetes Federation. 2015.

4. Bhandari GP, Angdembe MR, Dhimal M, Neupane S, Bhusal C. State of non-communicable diseases in Nepal. BMC public health. 2014 Dec;14(1):23.

5. Mailloux, Lionel. "Dialysis in diabetic nephropathy".2007.

6. Yau JW, Rogers SL, Kawasaki R, Lamoureux, EL, Kowalski JW, Bek T, ... \& Haffner, S.. Global prevalence and major risk factors of diabetic retinopathy. Diabetes care. 2012 35(3), 556-564.

7. Naheed T, Khan A, Masood G, Yunus BB, Chaudry MA. Dyslipidemias in type II diabetes mellitus patients in a teaching hospital of Lahore, Pakistan. Pakistan Journal of Medical Sciences. 2003;19(4):283-6.

8. Kalofoutis C, Piperi C, Kalofoutis A, Harris F, Phoenix D, Singh J. Type II diabetes mellitus and cardiovascular risk factors: current therapeutic approaches. Experimental \& Clinical Cardiology. 2007;12(1):17.

9. Chudyk A, Petrella RJ. Effects of exercise on cardiovascular risk factors in type 2 diabetes: a meta-analysis. Diabetes care. 2011 May 1;34(5):1228-37.

10. Wu L, and Parhofer KG. "Diabetic dyslipidemia." Metabolism-Clinical and Experimental. 2014, 63(12): 14691479.

11. Nathan DM, Cleary PA, Backlund JY, et. al. Intensive diabetes treatment and tcardiovascular disease in patients with type 1 diabetes". The New England Journal of Medicine. 2005. 353 (25): 2643-53.

12. Organization, WH. "Definition and diagnosis of diabetes mellitus and intermediate hyperglycaemia": report of a WHO/IDF consultation. 2006.

13. Vazquez-Benitez G, Desai JR, Xu S, Goodrich GK, Schroeder EB, Nichols GA, Segal J, Butler MG, Karter AJ, Steiner JF, Newton KM. Preventable major cardiovascular events associated with uncontrolled glucose, blood pressure, and lipids and active smoking in adults with diabetes with and without cardiovascular disease: a contemporary analysis. Diabetes Care. 2015 May 1;38(5):905-12. 